\title{
ESTATUTOS DE AUTONOMÍA Y SISTEMAS ELECTORALES AUTONÓMICOS*
}

\author{
LUIS A. GÁLVEZ MUÑOZ \\ Catedrático de Derecho Constitucional \\ Universidad de Murcia
}

TRC, núm. 45, 2020, pp. 325-349

ISSN 1139-5583

\section{SUMARIO}

I. Introducción. II. El sistema electoral en los Estatutos. III. La importancia de las regulaciones establecidas. IV. Las principales innovaciones aportadas. V. Valoración de la regulación. VI. Breve referencia a la jurisprudencia constitucional.

\section{INTRODUCCIÓN}

El Derecho electoral y el Derecho autonómico son dos sectores fundamentales del actual Derecho constitucional español, como es propio de un Estado que se define como Democrático en el frontispicio de la misma Constitución (artículo 1.1 CE) y que se configura como Autonómico, tras el ejercicio por todas las nacionalidades y regiones de su derecho a la autonomía (art. 2 CE). El primero, el Derecho electoral, articula la representación, que es la vía ordinaria de participación política en toda democracia, y el segundo, el Derecho autonómico, es la expresión del ejercicio de las competencias asumidas por cada comunidad autónoma (CA).

Su confluencia se produce, con toda lógica, en el mismo texto constitucional, bien de forma expresa (art. 152.1, que establece algunas prescripciones sobre la elección de la asamblea legislativa de las comunidades autónomas, CCAA, de vía rápida), bien de manera implícita (artículo 147.2.c, que señala que los estatutos de autonomía, EEAA, deben regular la organización de sus instituciones de

* Sirva este artículo como homenaje a mi querido amigo y colega Mariano García Canales, Catedrático de Derecho Constitucional de la Universidad de Murcia, recientemente fallecido. Era, además de un excelente constitucionalista, una magnífica persona, de inolvidable recuerdo. 
autogobierno), bien incluso de modo indirecto, en las disposiciones más generales aplicables en materia electoral (artículos 23.1 y 81.2 e incluso $\left.149.1 .1^{\circ}\right)^{1}$. Y se produce también, como es natural, en una pluralidad de normas, que, de una u otra forma, se refieren a las elecciones autonómicas: desde la Ley Orgánica 5/1985, de 19 de junio, del Régimen Electoral General (LOREG), por medio de su disposición adicional primera, 2, a los decretos del presidente de la CA respectiva relativos a la convocatoria de las elecciones, pasando por los EEAA, las leyes electorales autonómicas e incluso las leyes del presidente y del gobierno de las CCAA y los reglamentos de las asambleas legislativas autonómicas.

En este trabajo nos vamos a centrar en los elementos fundamentales de esta confluencia: el estatuto de autonomía (EA) y el sistema electoral. El EA, en tanto norma institucional básica de la CA llamada por la CE a establecer la organización de las instituciones de autogobierno, entre las que se encuentran las relativas a la elección de la asamblea legislativa; y, el sistema electoral, en cuanto elemento clave de la regulación de toda elección, al ocuparse, a través de sus distintas piezas, de estructurar el voto de los ciudadanos y realizar la traducción de tales votos en escaños ${ }^{2}$. Nos proponemos, en particular, conocer lo que señalan los EEAA sobre el sistema electoral, calibrar su importancia y determinar sus aportaciones al conjunto del Derecho electoral español, para concluir, finalmente, con una valoración final y una breve referencia a la jurisprudencia constitucional recaída sobre los escasos conflictos planteados.

\section{EL SISTEMA ELECTORAL EN LOS ESTATUTOS}

¿Qué establecen los EEAA sobre el sistema electoral relativo a las elecciones a las asambleas legislativas respectivas? ¿Cómo han regulado esta materia? Es la primera cuestión que debemos abordar, pues nos va a servir como apoyo para responder a las demas tareas planteadas. Vamos a verlo, poniendo de relieve tanto las prescripciones o declaraciones generales sobre la configuración del sistema, como las referencias a las distintas piezas que integran ese conjunto denominado sistema electoral, y que están estrechamente conectadas, como indica su propio nombre ${ }^{3}$.

a) Todos los EEAA han coincidido en declarar la adopción de la representación proporcional como base del sistema electoral. La aplicación de este modelo era obligada para los EEAA adoptados por la vía del artículo 151.2 de la CE (País Vasco, Cataluña, Galicia y Andalucía), a la vista del artículo 152.1, pero en el momento en que se redactaron los EEAA, a principios de los años 80 del pasado

1 Vid. Oliver Araujo, J., Los sistemas electorales autonómicos, Barcelona: IEA, 2011, pp. 54-58.

2 Sobre este concepto vid. NoHLEN, D., Sistemas electorales del mundo, Madrid: CEC, 1981, pp. 55-56; y RAE, D. W., Leyes electorales y sistemas de partidos, Madrid: CITEP, 1977, p. 11.

3 Vid. RAE, D. W., Leyes electorales..., op. cit., pp. 14-15. 
siglo, no estaba nada clara la exigencia de su aplicación en los EEAA que siguieron la vía ordinaria de aprobación prevista en el artículo 143.2 de la CE, cuestión que, como es sabido, aclaró el TC años después, en $1998^{4}$, aunque con amplia división doctrinal ${ }^{5}$.

El caso es que, sin existir formalmente la exigencia del sistema de representación proporcional para los EEAA de vía ordinaria, todos se ajustaron a ella, aunque no lo hicieron de forma espontánea o porque sí, sino siguiendo las recomendaciones de los Acuerdos Autonómicos de 31 de julio de 1981 entre el Gobierno de la Nación y el partido que lo sustentaba, Unión de Centro Democrático, con el principal partido de oposición entonces, el Partido Socialista Obrero Español. No se afirma expresamente esta opción en el texto, pero se da por asumida, dado el conjunto de sus prescripciones sobre la circunscripción electoral ${ }^{6}$.

Además, conviene precisar que las declaraciones estatutarias sobre la proporcionalidad que han de inspirar el sistema electoral siguen siendo válidas en el momento actual, tras la jurisprudencia del TC, pues, ésta podría cambiar o matizarse en el futuro; no es probable, pero tampoco descartable ${ }^{7}$. Incluso cobrarían nueva fuerza o podría eliminarse en caso de reforma constitucional.

b) El otro mandato implícito en el artículo 152.1 de la CE, el de asegurar la representación de las diversas zonas del territorio de la CA, no ha tenido el mismo éxito en su acogida formal, en la medida en que ha sido establecido explícitamente como tal declaración general por algo menos de la mitad de los EEAA: Aragón, Islas Baleares, Castilla-La Mancha, Castilla y León, Cataluña, Galicia y Valencia (aunque éste habla como posibilidad de la comarcalización) ${ }^{8}$. Además, todos ellos, salvo el EA de Cataluña, contienen, como veremos, alguna medida concreta de aplicación del mismo.

No obstante, es preciso indicar que hay otros EEAA que, sin hacer esta declaración, sí contienen, en cambio, algún elemento que trata de hacer realidad esa representación territorial, como podremos comprobar seguidamente. Es el caso de los EEAA del País Vasco, Islas Baleares, Canarias y Extremadura. Solo quedarían fuera de toda mención o aplicación de esta prescripción los EEAA de las

4 Fue la STC 225/1998, de 23 de noviembre, posteriormente reiterada en otras como las SSTC 19/2011, de 3 de marzo; 197/2014, de 4 de diciembre, y 15/2015, de 5 de febrero.

5 Sobre las dos posiciones mantenidas vid. Garrorena Morales, A., «Tribunal Constitucional y sistema electoral de las Comunidades Autónomas. Una desafortunada jurisprudencia», en Gálvez Muñoz, L. A (dir.), El Derecho electoral de las Comunidades Autónomas. Revisión y mejora, Madrid: CEPC, 2009, pp. 45-78; y Oliver Araujo, J., Los sistemas electorales autonómicos..., op. cit., pp. 35-46.

6 Vid. Presidencia del Gobierno, Acuerdos Autonómicos 1981, Madrid: Servicio de Publicaciones de la Presidencia del Gobierno, colección Informe, n 36, octubre de 1981, pp. 7-8.

7 Garrorena Morales, A., apunta en este sentido («Tribunal Constitucional y...», op. cit., pp. 73-76).

8 El artículo 23.2 del EA de Valencia dice que la elección se realizará en la forma que determina la Ley Electoral Valenciana «atendiendo a criterios de proporcionalidad y, en su caso, de comarcalización». 
CCAA uniprovinciales, a excepción de Islas Baleares, esto es, los de Murcia, Navarra, Madrid, Cantabria, Asturias y La Rioja.

En definitiva, buena parte de los EEAA, hagan o no la declaración de que ha de asegurarse la representación territorial en la elección, la aplican en mayor o menor grado. Y, entre ellos, los que lo hacen de forma más intensa son los EEAA de País Vasco, Castilla y León, Valencia, Andalucía y Extremadura.

c) Casi todos los EEAA — todos, salvo los de País Vasco e Islas Baleareshan abordado también la relevante cuestión del tamaño del Parlamento. No lo han hecho, sin embargo, de la misma manera, pues varían en su margen de precisión, que cabe clasificar en pleno, medio o reducido?.

En la mayoría de los EEAA el margen de concreción es medio, pues han establecido — como hace la CE con el Congreso de los Diputados ${ }^{10}$ — una horquilla de escaños en la que deberá moverse la decisión final del legislador (Asturias, Cantabria, La Rioja, Murcia, Aragón, Castilla-La Mancha $)^{11}$, pero hay otros modelos más singulares: en dos EEAA la precisión es plena (Madrid y Castilla y León $)^{12}$, pues se ha establecido la determinación automática del número de escaños de la Asamblea en función de los cambios de población; y en otros tres es baja, ya que solo se establece la cifra inferior de escaños que ha de tener el Parlamento (Andalucía y Valencia) ${ }^{13}$ o la superior (Extremadura) ${ }^{14}$.

d) Otra cuestión importante regulada en los EEAA de forma generalizada es la del tipo de circunscripción. Solo hay tres EEAA - Asturias, Navarra y Cataluña- que no dicen nada sobre el tema ${ }^{15}$, si bien en el caso del EA de Cataluña hay que matizar que sigue en vigor todavía, en defecto de ley electoral propia, la disposición transitoria cuarta del EA de 1979 que establece la circunscripción provincial $^{16}$. Además, hay otro EA, el de Murcia, que cabe equiparar a estas excepciones, pues la referencia que hace a la circunscripción es abierta y, por

9 Vid. Gálvez Muñoz, L. A., «Comentario al artículo 24. Elecciones a la Asamblea Regional», en Garrorena Morales, Á. y García Canales, M. (eds.), Comentarios al Estatuto de Autonomía de la Región de Murcia, Murcia: Asamblea Regional de Murcia et alii, 2005, pp. 1019-1020.

10 Artículo 68.1 de la CE.

11 Como ejemplo, el EA de Asturias establece que por ley se fijará el número de miembros del Parlamento «entre 35 y 45 » (art. 25.2).

12 Por ejemplo, el EA de Madrid determina en su artículo 10.2 que «la Asamblea estará compuesta por un Diputado por cada 50.000 habitantes o fracción superior a 25.000, de acuerdo con los datos actualizados del censo de población».

13 Así, el Estatuto de Andalucía señala que el Parlamento está constituido "por un mínimo de 109 diputados» (art. 101.1).

14 El EA de Extremadura señala que «los diputados de la Asamblea de Extremadura, en número máximo de 65, serán elegidos por sufragio universal....» (art. 17.1).

15 El EA de Islas Baleares también constituía una excepción en su versión inicial y hasta la reforma de 2007, que consagró las islas de Mallorca, Menorca, Ibiza y Formentera como circunscripciones. Y también lo fue el EA de Valencia hasta la reforma de 2006, que consagró la circunscripción provincial.

16 Esta disposición transitoria cuarta del EA de 1979 ha sido declarada vigente por la disposición transitoria segunda del EA de 2006. 
tanto, meramente retórica, al señalar que la ley fijará «la circunscripción o circunscripciones» ${ }^{17}$.

La mayoría de los EEAA han fijado, pues, en su articulado el tipo de circunscripción, y, además, casi todos ellos han optado por la provincia —incluidos los EEAA de las CCAA uniprovinciales de Madrid, La Rioja y Cantabria-. La única excepción es el EA de Islas Baleares, que ha identificado las islas como circunscripción electoral $^{18}$.

Caso especial, de difícil ubicación, por las dificultades de interpretación que presenta, es el del EA de Canarias, tras la reciente reforma de diciembre de 2018. La mención que hace a la circunscripción en su artículo 39.2.c) no cierra la cuestión, pero contiene algunas indicaciones precisas. Señala que «las circunscripciones electorales podrán ser de ámbito autonómico, insular o de ambas» y, al propio tiempo, sin solución de continuidad, indica que «cada una de las islas de El Hierro, Fuerteventura, Gran Canaria, La Gomera, Lanzarote, La Palma y Tenerife, constituyen una circunscripción electoral». La interpretación más razonable es considerar que el EA habilita tres alternativas, sin decantarse por ninguna de ellas (circunscripción única autonómica, circunscripción múltiple insular y combinación de circunscripciones autonómica e insulares) y que, además, para el caso de que operen las circunscripciones insulares, determina cuáles son éstas ${ }^{19}$. Por otra parte, conviene indicar que hasta tanto se cierre esta cuestión por la ley electoral, se aplica la disposición transitoria primera, 1, del EA, que consagra la doble circunscripción autonómica e insular ${ }^{20}$.

e) Lo que ya no está tan extendida es la regulación del prorrateo o reparto de escaños entre circunscripciones, aunque el número de EEAA que de alguna forma se ocupan de ella es elevado: casi la mitad de los EEAA (Castilla y León, Valencia, Extremadura, País Vasco, Andalucía, Aragón y Canarias ${ }^{21}$. Además, fuera de esta enumeración, hay que tener en cuenta, la regulación provisional del EA de Cataluña, que hace directamente el reparto de escaños, $y$, hasta hace unos pocos años, el EA de Castilla-La Mancha, que, sometido en este terreno a una fuerte división política, garantizó en su articulado a cada circunscripción, como mínimo, los escaños que hasta ese momento les atribuía la ley electoral ${ }^{22}$.

17 Artículo 24.2 del EA de Murcia.

18 Artículo 41.3 del EA de Islas Baleares: «Las circunscripciones electorales son las de Mallorca, Menorca, Ibiza y Formentera».

19 En este mismo sentido vid. López Aguilar, J. F. y García Mahamut, R., «El nuevo Estatuto de Autonomía de Canarias: «tercera generación», hecho diferencial y nuevo sistema electoral», REDC, $\mathrm{n}^{\circ} 115$, 2019, pp. 37-38.

20 La disposición transitoria primera, punto 1, del EA de Canarias, no es la original del EA de 1982, sino que ha recibido una nueva e importante redacción con el EA de diciembre de 2018.

21 El EA de Canarias contiene prescripciones al respecto tanto en el artículo 39 (punto 2.e), como, sobre todo, en la disposición transitoria primera, 2 (punto 1).

22 Artículo 10.2 del EA de Castilla-La Mancha, en la redacción dada por la Ley Orgánica 3/1997, de 3 de julio, y que estuvo vigente hasta 2014 (Ley Orgánica 2/2014, de 21 de mayo). 
Los EEAA que en la actualidad regulan esta cuestión en su articulado la abordan, sin embargo, de muy distinto grado y forma. La regulación más intensa es la del EA del País Vasco, que establece un mandato de reparto igualitario de escaños entre las tres circunscripciones ${ }^{23}$. Le sigue el EA de Valencia, que ha establecido tres prescripciones: la garantía inicial de un mínimo de veinte escaños para cada una de los tres distritos electorales en un Parlamento de al menos 99 escaños, el reparto en función de la población del resto de los escaños y un límite a la distorsión del valor población en el reparto de escaños, en concreto que «la desproporción que establezca el sistema resultante sea inferior a la relación de uno a tres» ${ }^{24}$, lo que supera en un cuarto de punto la recomendación de máxima corrección territorial contenida en los acuerdos autonómicos de $1981^{25}$. Y luego viene el EA de Castilla y León, que combina un mínimo provincial inicial de tres escaños con la asignación de un escaño más a cada circunscripción «por cada 45.000 habitantes o fracción superior a $22.500 »^{26}$.

Los demás EEAA tienen una densidad normativa menor en este terreno. En función del tipo de prescripción que realizan cabe distinguir dos grupos: de un lado, el EA de Extremadura, que ordena al legislador electoral establecer un mínimo provincial inicial antes de proceder al reparto de escaños en proporción a la población ${ }^{27}$; y los de Aragón, Canarias y Andalucía, que establecen límites a la acción legislativa o a su resultado en la distribución de escaños. Así, el EA de Aragón, señala que «la cifra de habitantes necesarios para asignar un diputado a la circunscripción más poblada no supere 2,75 veces la correspondiente a la menos poblada $»^{28}$; el de Canarias, que «a ninguna circunscripción insular se le podrá asignar un número de diputados y diputadas inferior a otra que tenga menos población de derecho» ${ }^{29}$, como prevención frente a la repetición de situaciones pasadas ${ }^{30} ;$ y el de Andalucía, que «ninguna provincia tendrá más del doble de Diputados que otra» ${ }^{31}$.

23 Artículo 26.1 del EA del País Vasco: «El Parlamento Vasco estará integrado por un número igual de representantes de cada Territorio Histórico...».

24 Artículo 24 del EA de Valencia: «La Ley Electoral Valenciana... garantizará un mínimo de 20 Diputados por cada circunscripción provincial, distribuyendo el resto del número total de los diputados entre dichas circunscripciones, según criterios de proporcionalidad respecto de la población, de manera que la desproporción que establezca el sistema resultante sea inferior a la relación de uno a tres». Para una interpretación distinta de este precepto vid. MARTínez Sospedra, M., «Sobre la necesidad de una ley electoral valenciana (los condicionantes constitucionales y estatutarios de la Ley Electoral», Corts. Anuario de Derecho Parlamentario, $\mathrm{n}^{\circ} 18,2007$, pp. 134-137.

25 Punto $5^{\circ}$ de los Acuerdos Autonómicos de 31 de julio de 1981, que señalaba que, con carácter general, «la corrección territorial entre las circunscripciones con menor y mayor censo electoral oscilará entre 1 a 1 y 1 a $2,75 \ll$. Vid. Presidencia del Gobierno, Acuerdos Autonómicos 1981, op. cit., p. 8.

26 Artículo 21.2 del EA de Castilla y León.

27 Artículo 17.3 del EA de Extremadura.

28 Artículo 36 del EA de Aragón.

29 Artículo 39.2.e) del EA de Canarias.

30 Vid. Fernández Esquer, C., «Algunos problemas en la articulación jurídica de los sistemas electorales autonómicos», Revista de las Cortes Generales, n 97-99, 2016, p. 343.

31 Artículo 104.1 del EA de Andalucía. 
f) Menos interés estatutario ha despertado el tema de la barrera electoral, que solo se contempla en tres EEAA (Madrid, Valencia y Canarias). El primero de ellos, el de Madrid, es el único que lo hace de forma plena, pues fija directamente la barrera «en el $5 \%$ de los sufragios válidamente emitidos» ${ }^{32}$. Los otros dos EEAA, los de Valencia y Canarias, se limitan a establecer el mandato de establecerla por $1 \mathrm{y}^{33}$, con la particularidad en este último caso que, en tanto no se fije en la ley, se aplica la establecida en la disposición transitoria primera, punto 2, del EA ${ }^{34}$.

Además, conviene recordar que, hasta la reforma de 2006, el EA de Valencia fijaba directamente la barrera ${ }^{35}$. Y, asimismo, que la regulación provisional del EA de Cataluña conduce, vía remisión normativa general, a la aplicación de la barrera del Congreso de los Diputados, esto es, la del $3 \%$ de los votos válidos emitidos en la circunscripción ${ }^{36}$.

\section{LA IMPORTANCIA DE LAS REGULACIONES ESTABLECIDAS}

Los EEAA realizan, en conjunto, una regulación reducida del sistema electoral autonómico, pero ello no supone necesariamente una tacha de irrelevancia o una merma de valor, pues en esta materia con muy poco se puede decir mucho; lo cuantitativo no se despliega, pues, como efecto reflejo, en lo cualitativo. De hecho, a la luz de los textos estatutarios, se puede decir que, efectivamente, la regulación que realizan sobre el sistema electoral de las asambleas legislativas territoriales tiene, en general, una importancia considerable, pues en buena parte de los casos las disposiciones que contienen configuran de manera muy notable su funcionamiento.

Basta echar un vistazo a algunas de las disposiciones estatutarias más comunes para darse cuenta de ello. Así, la asunción del sistema de representación proporcional, con postergación de los sistemas mayoritarios, y con clara incidencia sobre la formación de gobiernos; la determinación del tamaño aproximado del parlamento, elemento clave para el desarrollo del trabajo parlamentario y para la operatividad del principio constitucional de proporcionalidad de la representación; o la especificación del tipo de circunscripción, en especial en las CCAA uniprovinciales, con clara incidencia tanto sobre el principio de proporcionalidad como sobre el de territorialidad.

Y piénsese, asimismo, en la trascendencia de algunos de los elementos singulares contenidos en determinados EEAA. Veamos unos pocos ejemplos muy ilustrativos, entre otros posibles:

32 Artículo 10.6 del EA de Madrid.

33 Vid. los artículos 23.2 del EA de Valencia y 39.2.d) del EA de Canarias.

34 Por su parte, la Ley electoral valenciana (Ley 1/1987, de 31 de marzo) la establece en el artículo 12.a).

35 En el EA de Valencia anterior a 2006 la barrera era del 5\% de los votos emitidos y operaba a nivel de toda la CA (art. 12.2).

36 Artículo 163.1.a) de la LOREG. 
- En el EA de Madrid el establecimiento de la barrera electoral en el 5\% de los sufragios válidamente emitidos ${ }^{37}$. Esta barrera, que es alta, supone, como es propio de su esencia, la limitación de las posibilidades de representación de las minorías y la consiguiente alteración de la dinámica de partidos. Además, como dado el tamaño de la Asamblea de Madrid es factible alcanzar la cuota de reparto con un reducido porcentaje de sufragios, ha tenido aplicación efectiva en algunas ocasiones. Así, Izquierda Unida quedó fuera de la Asamblea en 2015 con un 4'14 \% de los votos, el Centro Democrático y Social en 1991 con el 3’39 \% y este partido también en 1983 con el 3'12\% ${ }^{38}$.

- En el EA del País Vasco la declaración de que las tres circunscripciones tienen el mismo número de escaños. Esta identidad de trato, cuando la población es cuantitativa y políticamente muy distinta en los tres territorios, produce evidentes distorsiones, y no solo en el valor del voto, que son muy elevadas, sino también en el ámbito político, y que puede afectar de manera importante a la relación entre votos y escaños de los distintos partidos $^{39}$; a cambio se pretende lograr una mayor integración de la propia CA. Como ejemplo real y extremo de distorsión cabe citar las elecciones autonómicas de 1986, donde un partido con menos votos que otro obtuvo más escaños que éste (PSOE frente a PNV) ${ }^{40}$.

- En el EA de Cataluña (regulación provisional) el reparto fijo de escaños entre las cuatro circunscripciones. Esta normativa no es, como cabe suponer, neutra — apreciación que es, precisamente, lo que explica su vigencia-, pues consagra un reparto de escaños entre las cuatro circunscripciones provinciales que no es proporcional a la población respectiva y que, además, cada vez está más desfasado, en claro perjuicio de las circunscripciones más pobladas, y que también son las que tienen un electorado menos nacionalista ${ }^{41}$. Su aplicación ha producido también graves resultados

37 Artículo 10.6 del EA de Madrid.

38 En las elecciones de 2019 Unidas Podemos pasó por poco la barrera, obteniendo el 5,5\% de los votos validos

39 Vizcaya tiene más del triple de población que Álava, y Guipúzcoa más del doble, y los tres territorios presentan una muy diferente adscripción política: en Vizcaya existe mayoría nacionalista y primacía clara del PNV, en Guipúzcoa la mayoría nacionalista es todavía más acentuada y con un alto porcentaje de votos para Bildu y en Álava, finalmente, hay una mayoría no nacionalista. Vid. los resultados de las distintas elecciones en la página web de la Junta Electoral Central: http://www.juntaelectoralcentral.es/cs/jec/elecciones/ autonomicas/paisvasco

40 El PSOE con 252.233 votos obtuvo diecinueve escaños y el PNV con 271.208 consiguió dos menos, diecisiete. Vid. los resultados en el Boletín Oficial del País Vasco de 4 de marzo de 1987, pp. 973 y ss.

41 Vid. López Carmona, A., Ramírez González, V. y Delgado Márquez, B., «Una propuesta de sistema electoral para Cataluña: reparto biproporcional», Política y Sociedad, vol. 51, nº 3, 2014, pp. 841 y ss.; Oliver Araujo, J., Los sistemas electorales autonómicos..., op. cit., pp. 89-91; y Fernández Esquer, C., «Algunos problemas en...», op. cit., pp. 350-352. 
paradójicos $^{42}$ : en las elecciones autonómicas de 1999 y de 2003 el Partido Socialista de Cataluña recibió más votos que Convergençia i Unió, pero menos escaños ${ }^{43}$.

- En el EA de Canarias (regulación provisional) el prorrateo de escaños entre las circunscripciones insulares, que, hasta 2018, era el único tipo de circunscripción previsto. El prorrateo, establecido de manera fija, ha estado siempre fuertemente sesgado, como consecuencia de la imposición política de la llamada «regla de las tres paridades» en número de escaños, esto es, paridad entre las dos provincias que integran la CA de Canarias (Las Palmas y Santa Cruz de Tenerife), paridad dentro de cada provincia entre el conjunto de las islas menores y la isla mayor (en la provincia de Las Palmas: Fuerteventura y Lanzarote frente a Gran Canaria; y en la de Santa Cruz de Tenerife: El Hierro, La Gomera y La Palma frente a Tenerife) y paridad entre el conjunto de las islas menores y el de las dos islas mayores (Las Palmas Fuerteventura, Lanzarote, El Hierro, La Gomera y La Palma frente a Gran Canaria y Tenerife ${ }^{44}$. Esta regla se ha atenuado mínimamente tras la última reforma estatutaria ${ }^{45}$, pero su anterior rigidez ha conducido históricamente a distorsiones descomunales, con las consecuencias jurídicas (igualdad, proporcionalidad), políticas (pluralismo, sistema político, formación de gobierno) y de todo tipo que cabe imaginar (redes clientelares, desmovilización ciudadana).

Tres datos de impacto del pasado reciente: el $83 \%$ de los electores (el de las dos islas mayores) eligen el mismo número de escaños (50\%) que el $17 \%$ restante (el del conjunto de las islas menores); dos, el voto de quienes viven en la isla menos poblada (El Hierro) ha valido diecisiete veces más que el de quienes residen en la isla con mayor número de habitantes (Tenerife); y tres, una isla con menos población que otra ha tenido, en cambio, más escaños (La Palma, 82.346 habitantes y 8 escaños, y Fuerteventura, 107.367 habitantes y 7 escaños) ${ }^{46}$.

42 Vid. Lago Peñas, F. J., y Montero Gibert, J. R., «Más votos y menos escaños: el impacto del sistema electoral en las elecciones autonómicas catalanas de 2003», Revista Española de Investigaciones Sociológicas, $\mathrm{n}^{\circ} 115,2004$, pp. 11-42.

43 Vid. los resultados de las distintas elecciones en la página web de la Generalitat: http://politiquesdigitals.gencat.cat/ca/pgov_ambits_d_actuacio/pgov_eleccions/pgov_dades_electorals/

44 Torres Del Moral, A., advierte, además, otras dos paridades: la paridad entre las dos islas mayores y la existente entre los conjuntos de islas periféricas de cada provincia ( «Sistemas electorales y sistemas de partidos en las Comunidades Autónomas», en Gálvez Muñoz, L. A. (dir.), El Derecho electoral de las Comunidades Autónomas. Revisión y mejora, Madrid: CEPC, 2009, p.234).

45 Se ha asignado un escaño más a la circunscripción insular de Fuerteventura.

46 Vid. López Aguilar, J. F. y García Mahamut, R., «El nuevo Estatuto...», op. cit., pp. 33-36; y FERNÁNDEZ ESQUeR, C. y MONTERo, J. R., «El sistema electoral canario: representación desproporcionada y desigualdad del voto», en Pérez SÁnchez, G. y Mujica Moreno, V. (coords.), Textos para la reforma del sistema electoral canario, Gran Canaria: Universidad de las Palmas de Gran Canaria, 2016, pp. 27-74. 
Dicho esto, es necesario realizar varias precisiones de interés, la última de ellas muy llamativa, como se comprobará. Veamos cuáles son.

a) La primera es que no todos los EEAA contienen el mismo nivel de regulación del sistema electoral autonómico. Hay EEAA que regulan esta materia con mayor detalle y otros que apuntan lo mínimo sobre el mismo. En el primer grupo cabe citar los EEAA de Valencia, Castilla y León y Canarias (en especial, si se complementa con la regulación provisional); y entre los segundos los de Murcia, Asturias Navarra y Cataluña.

Esta diferencia entre EEAA puede tener múltiples causas. Una de ellas es, sin duda, la de la coyuntura política de cada CA, pues es comprensible que los partidos políticos incorporen al EA los aspectos en los que están de acuerdo o en los que logran un acuerdo con cesiones mutuas, dejando para el legislador la concreción de los elementos más problemáticos y conflictivos. Otras posibles causas, apuntadas muy brevemente, podrían ser el concepto de EA que se tenga mayoritariamente en una CA, la confianza en las posibilidades de lograr acuerdos posteriores con otras coyunturas o mayorías o el deseo de eludir en la configuración del sistema el control de las Cortes Generales, al ser el EA una ley orgánica, aunque de origen y tramitación especial.

b) La segunda precisión consiste en advertir que los EEAA han dejado, con todo, un amplio grado de libertad a las asambleas legislativas de las CCAA para regular el sistema electoral autonómico, respetando, naturalmente, sus disposiciones. La regulación estatutaria es relevante, como hemos dicho, pero no completa, ni exhaustiva.

Las CCAA disponen así, por lo general, por vía de las leyes electorales que aprueben sus respectivos parlamentos, de una amplia facultad de decisión en los diversos aspectos del sistema electoral ${ }^{47}$. Así, todas tienen plena libertad para decidir el tipo de listas electorales (cerradas y bloqueadas, cerradas y desbloqueadas o abiertas $)^{48}$, concretar la fórmula electoral a utilizar en el ámbito de las proporcionales $^{49}$ o introducir mecanismos de igualación o corrección de los efectos del sistema aparte de la barrera ${ }^{50}$; y casi todas disponen de capacidad para establecer o no una barrera electoral ${ }^{51}$, determinar el porcentaje de la barrera y su ámbito,

47 El margen de libertad del legislador autonómico no es, obviamente, el mismo en todas las CCAA, sino que es mayor o menor en función del grado de concreción al que ha llegado cada EA en esta materia, como hemos visto en el punto anterior.

48 Vid. Fernández Esquer, C. y Sierra Rodríguez, J., «El voto preferencial en las Comunidades Autónomas: propuestas y condicionantes jurídicos», Revista d'estudis autonòmics i federals, n 29, 2019, pp. 51-100.

49 Sobre las distintas fórmulas proporcionales vid. Nohlen, D., Sistemas electorales del mundo, op., cit., pp. 127 y ss.; y Martínez Sospreda, M., Marco Marco, J. y Uribe Otalora, A., Sistemas electorales. Un estudio comparado, Valencia: Tirant lo Blanch, 2007, pp. 130 y ss.

50 Vid., por ejemplo, Martínez Sospreda, M., Marco Marco, J. y Uribe Otalora, A., Sistemas electorales..., op. cit., pp. 93 y ss.

51 Las excepciones son las CCAA de Madrid, Valencia y Canarias. En la primera, como sabemos, la barrera electoral la establece el EA, mientras que en las otras dos los EEAA imponen su previsión al legislador. 
concretar el tamaño concreto del Parlamento, dentro del margen que dejan habitualmente los EEAA ${ }^{52}$, e, incluso, en menor medida, realizar el reparto de escaños entre las circunscripciones ${ }^{53}$.

c) La última precisión, que hemos avanzado que iba a resultar llamativa, nos es, sin embargo, parcialmente conocida. Hace referencia a las relevantes consecuencias que puede tener la escasa regulación del sistema electoral en el EA; y es que si, tras la elaboración del EA, las fuerzas políticas no se ponen de acuerdo en la aprobación de la ley electoral o en el establecimiento en la misma del sistema electoral, se aplicarían directamente las disposiciones provisionales que al respecto contuviera el propio EA o, llegado el caso, incluso las disposiciones previstas en la normativa electoral estatal para el Congreso de los Diputados ${ }^{54}$.

Esta situación se ha producido en dos CCAA: Cataluña y Canarias. En Cataluña la incapacidad para aprobar la ley electoral propia desde hace más de cuarenta años ha determinado la activación en todos los procesos electorales de la disposición transitoria cuarta del EA de 1979, declarada vigente por la disposición transitoria segunda del EA de 200655. En ella se establece el número de diputados del Parlamento, las circunscripciones y el reparto de escaños entre ellas, además de declarar la aplicación «de las normas vigentes para las elecciones al Congreso de los Diputados de las Cortes Generales» ${ }^{56}$, lo que afecta, entre otras cuestiones, a la barrera electoral ${ }^{57}$.

En Canarias, por su parte, la situación ha sido algo menos intensa que en Cataluña, pues ha bastado con la regulación contenida en su articulado (en distintos preceptos a lo largo del tiempo) y en la disposición transitoria primera del $\mathrm{EA}^{58}$, sin acudir a la legislación electoral sobre el Congreso de los Diputados. Las dos disposiciones sufrieron una importante reforma de sus contenidos en 1996 y recientemente, en 2018, con el nuevo EA, otra aún más relevante ${ }^{59}$.

52 Hay excepciones, como hemos visto: Castilla y León y Madrid, cuyos EEAA agotan la regulación del tamaño del Parlamento.

53 No en todas las CCAA. En el País Vasco el EA consigna el reparto igualitario entre las circunscripciones y en Castilla y León y Valencia los EEAA dejan un margen reducido para el prorrateo.

54 Desde 1985, el Título II de la LOREG. Con anterioridad, el Real Decreto Ley 20/1977, de 18 de marzo, sobre normas electorales.

55 Sobre el sistema electoral de Cataluña y la falta de ley electoral vid. OLIVER Araujo, J., Los sistemas electorales autonómicos..., op. cit., pp. 89-91.

56 El número 5 de la disposición transitoria cuarta del EA de Cataluña de 1979 dice: «En todo aquello que no esté previsto en la presente disposición serán de aplicación las normas vigentes para las elecciones legislativas al Congreso de los Diputados de las Cortes Generales».

57 Así lo confirmó la STC 75/1985, de 21 de junio, Fj 3.

58 En el texto primigenio del EA el artículo que regulaba las elecciones autonómicas fue el 8, con la reforma de 1996 pasó a ser el 9 y hoy, con el nuevo EA de 2018, es el 39. La regulación provisional siempre ha estado en la disposición transitoria primera, tanto del EA de 1982, como en el actual.

59 Sobre la regulación provisional del EA de Canarias vid. López Aguilar, J. F. y García MaHAmut, R., «El nuevo Estatuto...», op. cit., pp. 33 y ss.; PÉREZ SÁNCHEZ, G. y MujICA Moreno, V. (coords.), Textos para la reforma del sistema electoral canario, Gran Canaria: Universidad de las Palmas de Gran Canaria, 2016; y López Aguilar, J. F., «Los problemas de constitucionalidad del sistema electoral autonómico canario. (La «insoportable permanencia» de una Disposición transitoria)», REDC, $\mathrm{n}^{\circ}$ 51, 1997, pp. 95-140. 


\section{LAS PRINCIPALES INNOVACIONES APORTADAS}

Si hay un ámbito proclive a la innovación electoral ese es sin duda el del sistema electoral autonómico La amplia capacidad de decisión en esta materia, dentro del respeto a las disposiciones constitucionales, el deseo de atender a las peculiaridades políticas, territoriales e institucionales de cada CA y el sentimiento innato de diferenciación en algunas de ellas, entre otros elementos, abonan el terreno para las originalidades, singularidades o innovaciones.

Esta posibilidad se ha plasmado efectivamente en los EEAA, más que en las leyes electorales ${ }^{60}$. No se trata, entiéndase bien, de que cada EA, individualmente considerado, suponga una obra de innovación, nada más lejos de la realidad, sino que el conjunto de la regulación estatutaria permite descubrir ciertas originalidades en relación con el sistema electoral de las demás elecciones, en especial las relativas al Congreso de los Diputados.

Desde esta perspectiva se detectan interesantes elementos de innovación en los EEAA. Los más relevantes son los siguientes:

- La determinación automática del tamaño del Parlamento en función de los cambios de población: los EEAA de Madrid y de Castilla y León ${ }^{61}$.

- La reducción del tamaño del Parlamento frente a la tendencia habitual de sentido contrario: EA de Castilla-La Mancha ${ }^{62}$.

- La circunscripción única: los EEAA de las CCAA uniprovinciales de Madrid, La Rioja y Cantabria ${ }^{63}$.

- La existencia de una doble circunscripción, una territorial y otra referida al conjunto de la CA, con doble reparto de escaños, e incluso, según se ha interpretado, doble lista electoral con doble urna ${ }^{64}$ : el actual EA de Canarias ${ }^{65}$.

- El establecimiento de un límite a la distorsión del valor población en el reparto de escaños entre circunscripciones que introduzca primas de territorialidad: el EA de Aragón, que establece la diferencia máxima en 1 a $2^{7} 75^{66}$; el de Valencia, que la fija en la relación 1 a $3^{67}$; y el de Canarias, que prohíbe que una circunscripción con más habitantes que otra pueda tener menos escaños ${ }^{68}$.

60 Vid. Gavara de CARA, J. C., La homogeneidad de los sistemas electoral autonómicos, Madrid: CEPC, 2007, p. 103. Sobre la escasa originalidad de las leyes electorales autonómicas vid. Oliver Araujo, J., Los sistemas electorales autonómicos..., op. cit., pp. 82-89.

61 Vid. los artículos 10.2 del EA de Madrid y 21.2 del EA de Castilla y León.

62 Vid. García Maнамut, R., «La reforma de los sistemas electorales autonómicos tras las elecciones de 24 de mayo de 2015: vectores políticos y jurídicos», Teoría y Realidad Constitucional, no 41, 2018, pp. 187-188.

63 Artículos 10.5 del EA de Madrid, 17.3 del EA de La Rioja y 10.2 del EA de Cantabria.

64 Vid. López Aguilar, J. F. y García Mahamut, R., «El nuevo Estatuto de...», op. cit., pp. 41 y ss.

65 Artículo 39.2.c) del EA de Canarias.

66 Artículo 36 del EA de Aragón.

67 Artículo 24 del EA de Valencia.

68 Artículo 39.2.e) del EA de Canarias. 
- En sentido contrario, la introducción de un límite a la diferencia de escaños entre circunscripciones que podría producir el reparto proporcional de escaños en función de la población: el EA de Andalucía, que fija la diferencia máxima en el doble de escaños ${ }^{69}$. Antes también lo hacía el EA de Castilla-La Mancha, entre 1997 y 2014, que garantizaba un mínimo particularizado de escaños a cada circunscripción, con independencia de lo que pudiera resultar del reparto por población ${ }^{70}$.

- El prorrateo rígido o permanente de escaños entre circunscripciones, sin atender a posibles variaciones futuras de población: el EA de País vasco, que asigna el mismo número de representantes a cada territorio ${ }^{71}$, así como las regulaciones transitorias de los EEAA de Cataluña y Canarias, aún en vigor, que atribuyen una cifra determinada de escaños a cada circunscripción.

- La previsión de una barrera electoral alternativa: la regulación provisional del EA de Canarias, que, en el caso de la circunscripción insular, exige obtener un porcentaje elevado de votos en la isla de que se trate (15\%) o, alternativamente, un porcentaje menor (4\%), pero en la totalidad de la $\mathrm{CA}^{72}$.

- La fijación de una barrera electoral supradistrito, es decir, que dirige a todo el territorio autonómico el porcentaje de votos a obtener como mínimo por una candidatura para poder entrar en el reparto de escaños: el EA de Valencia antes de la reforma de $2006^{73}$, y la regulación provisional del EA de Canarias, que, como acabamos de ver, contempla, para la circunscripción insular, una barrera autonómica como alternativa a la específicamente establecida para ella ${ }^{74}$.

- El establecimiento de una barrera electoral elevada: no nos referimos a los EEAA que la fijan o la han fijado en el pasado en el $5 \%$ de los votos válidos emitidos $^{75}$, como hace también la LOREG con las elecciones locales ${ }^{76}$, sino al caso particular del EA de Canarias, que siempre ha contemplado barreras elevadas, y en la actualidad también, aunque más baja ahora que en el pasado: la regulación provisional actual establece la barrera, en el caso de las

69 Artículo 104.1 del EA de Andalucía.

70 El artículo 10.2 del EA de Castilla-La Mancha, en la redacción dada por la Ley Orgánica 3/1997, de 3 de julio, tras señalar que las Cortes de Castilla-La Mancha estarán constituidas por un mínimo de 47 Diputados y un máximo de 59, decía, a modo de garantía territorial, que «la asignación de Diputados a cada provincia no será inferior a la actual».

71 Artículo 26.1 del EA del País Vasco.

72 Disposición transitoria primera, punto 2, del EA de Canarias. Es una disposición tradicional en este EA: antes lo hizo el art. 8, vigente entre 1982 y 1996; y después, la disposición transitoria primera, punto 2, en la redacción vigente desde la reforma de 1996 a la de 2018.

73 Vid. el artículo 12.2 del EA de Valencia antes de la reforma de 2006.

74 Disposición transitoria primera, punto 2, del EA de Canarias. También contemplaron barreras supradistrito las anteriores regulaciones del sistema electoral en el EA de Canarias, tanto en el articulado al principio (artículo 8.2 del EA inicial), como en la disposición transitoria después (reforma de 1996).

75 El EA de Madrid en la actualidad y el de Valencia hasta la reforma de 2006.

76 Artículo 180 de la LOREG. 
circunscripciones insulares, en el $15 \%$ de los votos válidos en la respectiva circunscripción o, alternativamente, en el $4 \%$ de los votos válidos en la totalidad de la CA; la regulación provisional vigente entre 1996 y 2018 exigía, bien mayoría de votos o $30 \%$ de votos en la circunscripción, bien $6 \%$ de votos en toda la comunidad; y la regulación inicial de 1982, prevista en el artículo 8.2 del EA, requería «el 3\% de los votos válidos emitidos en la Región o el $20 \%$ de los válidamente emitidos en la respectiva circunscripción electoral» ${ }^{77}$.

- La determinación de que el cómputo de la barrera se hace no sobre el total de votos válidos emitidos, sino, simplemente sobre los «emitidos», comprendiendo tanto los votos a candidaturas y en blanco, como los nulos, lo cual aumenta, aunque levemente, la dificultad de su superación ${ }^{78}$ : el EA de Valencia hasta su reforma en $2006^{79}$. $^{\circ}$

- Finalmente, la exigencia de una mayoría cualificada para aprobar los preceptos legales que regulen el sistema electoral, frente a las habituales exigencias de rigidez para toda la ley electoral ${ }^{80}$ : regulación provisional de EA de Canarias $^{81}$, hasta la reforma de 2018 en que se adoptó el régimen habitual ${ }^{82}$.

Por otra parte, además de los elementos de innovación sobre el sistema electoral actualmente presentes en los EEAA, hay otros muchos que podrían introducirse en el futuro. No nos referimos a sugerencias doctrinales, sino a propuestas más o menos formales realizadas con ocasión de los procesos de reforma electoral que se han puesto en marcha en muchas CCAA en los últimos años, especialmente desde el famoso y ya lejano movimiento del «15-M» (de 2011). Aunque mayoritariamente las propuestas se dirigen a la reforma de las leyes electorales autonómicas, muchas son susceptibles de encaje estatutario y así se ha planteado en ocasiones ${ }^{83}$.

No es el momento de abordar el examen de estos elementos, pero sí conviene al menos citar los de mayor significación en el ámbito estatutario. Así:

- Elección de una parte de los diputados en distritos uninominales a través de un sistema mayoritario. El resto se seguirían eligiendo mediante sufragio de lista y fórmula proporcional en las circunscripciones actuales.

- Establecimiento de una circunscripción autonómica específica con unos pocos escaños para realizar el reparto de los restos que deriven del uso de la fórmula d'hondt en las circunscripciones ordinarias.

77 Sobre la regulación anterior de la barrera en Canarias vid., entre los muchos trabajos publicados, Oliver Araujo, J., Las barreras electorales. Gobernabilidad versus representatividad, Valencia: Tirant lo Blanch, 2017, pp. 134-138.

78 Vid. Oliver Araujo, J., Las barreras electorales..., op. cit., pp. 130-133.

79 Artículo 12.2 del EA de Valencia inicial.

80 Vid. Garrorena Morales, A., «Reforma y rigidez de la ley electoral en las Comunidades Autónomas», en Biglino Campos, P. (ed.), Nuevas expectativas democráticas y elecciones, Madrid: Iustel, 2008, pp. 35-38.

81 Se exigía una mayoría de 2/3 en el Parlamento para su aprobación (disposición adicional primera, 1).

82 La reforma de 2018 del EA de Canarias bajó, además, la mayoría requerida a 3/5 (art. 39.2).

83 García Mahamut, R., «La reforma de...», op. cit., pp. 196, 202 y 208. 
- Introducción de un sistema de listas flexibles o desbloqueadas. La teoría ofrece una amplia variedad de opciones ${ }^{84}$, pero parece imponerse la idea de limitar el número de preferencias electorales a disposición del elector y de exigir la obtención de un determinado porcentaje de preferencias para ser elegido «de manera preferente» y no por el orden de lista.

- Imposición a los partidos de la exigencia de que las listas que presenten tengan una composición plural desde el punto de vista de la procedencia o arraigo territorial de los candidatos. Su concreción adolece de cierta complejidad y puede tener difícil compatibilidad con el desbloqueo de listas.

\section{VALORACIÓN DE LA REGULACIÓN}

Tras conocer el contenido de la regulación de los EEAA sobre el sistema electoral de las elecciones autonómicas, determinar su relevancia y advertir sus singularidades o innovaciones en relación con el conjunto de las normas electorales del país, en especial las del Congreso de los Diputados, parece oportuno completar el estudio con dos consideraciones adicionales. Una se dirige a expresar la valoración que nos merece y la otra a dar cuenta de la jurisprudencia del TC recaída sobre ella.

De la primera nos ocupamos ahora. A este fin, hay que comenzar por señalar que nuestra valoración de la regulación realizada por los EEAA de los sistemas electorales autonómicos es, en términos generales, moderadamente positiva. Estamos de acuerdo, en primer lugar, con la decisión mayoritaria de los legisladores de regular en el EA diversas cuestiones del sistema electoral, así como con el grado de densidad normativa, pues ello resulta coherente con la importancia de la materia. Estamos también de acuerdo, en segundo término, con la introducción de medidas de innovación en muchos EEAA, con el fin de responder a las singularidades propias o deseos de lograr la mejor representación, por ser legítimo y ajustado al concepto de autonomía política. Y estamos, por último, de acuerdo con varias de las medidas más frecuentes en el panorama estatutario. Así, entre otros, los mandatos de proporcionalidad y territorialidad, que, nos parecen razonables y respecto de los que conviene precisar que no incurren en la tacha de la lex repetita ${ }^{85}$, pues ésta no opera propiamente en la relación entre la CE y los EEAA, sino solo en la del Estado

84 Vid. Simón, P., «Es hora de las listas desbloqueadas», Politikon, 1 de abril de 2014, disponible en https://politikon.es/2014/04/01/es-hora-de-las-listas-desbloqueadas/

85 Se suele usar esta expresión (lex repetita) para referirse a los casos de reproducción de normas estatales por leyes autonómicas. Esta técnica, además de constituir una deficiente técnica legislativa, incurre en inconstitucionalidad cuando, según ha señalado el TC, «la reproducción normativa se concreta en normas relativas a materias en las que la Comunidad Autónoma carece de competencia» (entre otras, SSTC 62/1991, de 22 de marzo, Fj 4 b); 147/1993, de 29 de abril, Fj 4, 341/2005, de 21 de diciembre, Fj 9, y 159/2016, de 22 de septiembre, $\mathrm{Fj}$ 3). 
y las CCAA y, además, como vimos, no los exige expresamente la CE para todas las CCAA $^{86}$; la utilización del sistema de la horquilla para establecer el tamaño del Parlamento, en la medida en que define suficientemente la cuestión sin cerrarla del todo; la designación de la provincia como circunscripción en todas las CCAA pluriprovinciales, salvo en Canarias, dada la inserción de esta división administrativa en el imaginario territorial español y que sirve eficazmente al cumplimiento del mandato de representación «de las diversas zonas del territorio»; o la no consagración en el EA de ciertas decisiones frecuentes en la normativa electoral española como la barrera electoral —excepción de Madrid — o las listas cerradas y bloqueadas, aunque tampoco es negativo que puedan estar si esa es la voluntad política mayoritaria en la CA.

Esto no significa, en modo alguno, que no tengamos discrepancias. Las tenemos y en gran número, cuestión que conviene exponer con algo más de detalle, a fin de poder contribuir mejor a las posibles reformas. Desde esta perspectiva son varias las críticas a realizar:

a) La escasa regulación del sistema electoral en los EEAA de algunas CCAA (Murcia, Asturias y Navarra), por las razones ya apuntadas de considerar al EA como la norma adecuada para trazar las bases de esta regulación tan relevante. En estos casos los EEAA se han limitado a sentar el criterio de representación proporcional, ya apuntado en el artículo 152.1 de la CE, y a establecer el tamaño aproximado del Parlamento.

Tampoco es muy expresivo el EA de Cataluña, que, a lo dicho por los otros, añade el mandato de aseguramiento de la representación de las distintas zonas de la comunidad (art. 56.1 y 2). No obstante, las fuerzas políticas no pudieron consensuar la concreción de este mandato en un tipo de circunscripción determinado, ante propuestas de diverso tipo: provincias, veguerías, circunscripción única o sistema mixto ${ }^{87}$.

b) El elevado tamaño del Parlamento previsto en los EEAA de muchas CCAA, sin justificación por el número de circunscripciones o la necesidad de dotar a la cámara de un mínimo tamaño representativo. Así, ante todo, el EA de Madrid, comunidad con circunscripción única y con un alto volumen de población, que fija un escaño por cada 50.000 habitantes o fracción superior a 25.000 (lo que da en la actualidad 129 escaños), y el de Valencia, «no inferior a 99» escaños (en la actualidad se fija en esta cifra $)^{88}$.

86 En otro sentido vid. Torres del Moral, A., «Sistemas electorales y...», op. cit., p. 220.

87 Vid. Sol Ordins, P., «Las instituciones de la Generalitat (I): El Parlamento de Cataluña», en Barceló Serramalera, M. y Vintró Castells, J. (coords.), Derecho Público de Cataluña, Barcelona: Atelier, 2008, pp 278-279.

88 Vid. el Decreto 2/2019, de 4 de marzo, del President de la Generalitat, de disolución de Les Corts y de convocatoria de elecciones a las mismas, de interés por ser la Ley electoral vigente anterior al nuevo EA y no haber sido actualizada. 
También cabe citar otros EEAA: el de Aragón (65-80 escaños para tres circunscripciones) y Extremadura (máximo 65 escaños para dos circunscripciones), e incluso los de las CCAA uniprovinciales de Murcia (45-55 escaños) y Navarra (40-60 escaños) si el tamaño del Parlamento no va acompañado luego, en la ley electoral, de circunscripciones infraprovinciales que lo justifiquen ${ }^{89}$.

Caso distinto es el del EA de Cataluña, que otorga un tamaño elevado al Parlamento (de 100 a 150 escaños), pero existe la posibilidad futura de que haya numerosas circunscripciones (como serían, por ejemplo, las veguerías); en cualquier caso, en la actualidad el Parlamento tiene 135 escaños para cuatro circunscripciones (regulación provisional del EA de 1979), lo que tiene difícil justificación. Más fundamentado parece el elevado tamaño del Parlamento andaluz fijado en el EA (un mínimo de 109 escaños), pues se trata de la comunidad más poblada de España con ocho provincias.

c) En sentido contrario, el reducido tamaño del Parlamento previsto en los EEAA de Castilla-La Mancha y Castilla y León. El caso más claro es el del actual EA de Castilla-La Mancha que, tras la reforma de 2014, ha fijado el número de diputados del Parlamento en la horquilla entre 25 y 35 para una división territorial con cinco circunscripciones, pues puede dar lugar, sobre todo si nos vamos a la línea de horquilla más baja de 25 escaños, a circunscripciones muy pequeñas, donde la proporcionalidad no puede funcionar bien; ocurre con todas las circunscripciones en general, pero, en especial, con las menos pobladas, las de Guadalajara y Cuenca, que, si se fijara el tamaño del Parlamento en la franja inferior, no superarían los tres escaños, abstracción hecha, evidentemente, de la posibilidad de que la ley electoral establezca mínimos provinciales iniciales ${ }^{90}$.

En cuanto al EA de Castilla y León, con otra forma de determinar el número de escaños, en función de la población, el resultado es el mismo. A pesar del mínimo provincial (de tres escaños) y que la cifra total de la Asamblea no es objetivamente baja (81 miembros), el elevado número de provincias (nueve), y las fuertes diferencias de población entre ellas, conduce inevitablemente a varias circunscripciones pequeñas. Así, en las elecciones de mayo de 2019 Soria tenía asignados cinco escaños y Segovia seis ${ }^{91}$.

d) Las reglas sobre la distribución o prorrateo de escaños que establecen los EEAA de algunas CCAA, debido a los fuertes desequilibrios del coste del escaño que producen entre algunas circunscripciones ${ }^{92}$, en perjuicio de la igualdad del voto

$89 \mathrm{Y}$, efectivamente, en estas dos CCAA no se prevén circunscripciones infraprovinciales. En Murcia las hubo hasta la reforma de Ley electoral de 2015 (Ley 14/2015, de 28 de julio).

90 La Ley electoral de Castilla-La Mancha contempla, efectivamente, un mínimo provincial inicial de tres escaños y, además, ha fijado el tamaño del Parlamento en treinta y tres miembros (artículo 16.1 y 2).

91 Vid. el artículo 2 del Decreto 1/2019, de 1 de abril, del Presidente de la Junta de Castilla y León, por el que se convocan elecciones a las Cortes de Castilla y León.

92 La doctrina habla también de prorrateo desviado o malapportionment, para referirse a la discrepancia entre el porcentaje de escaños que tiene asignados una circunscripción y el porcentaje de población que reside en la misma. En consonancia con ello se habla también del «índice de malapportionment» de un sistema 
de los ciudadanos, el mandato de proporcionalidad e incluso el derecho de sufragio pasivo de los contendientes, pues las distorsiones en la proporcionalidad, como ha apuntado el TC, pueden llegar a lesionarlo ${ }^{93}$. Los casos más pronunciados son los del País Vasco (distribución igualitaria de escaños) y Valencia (mínimo provincial elevadísimo de veinte escaños) que dan unas cifras de sesgo en el valor del voto o ratio de desigualdad de 1 a 3,6 en el primer caso (entre Álava y Vizcaya), y de 1 a 2,6 en el segundo (entre Castellón y Valencia). También son elevadas las distorsiones, de casi 1 a 2, que establecen los EEAA de Andalucía ${ }^{94}$ y Castilla y León (entre Huelva y Sevilla, y entre Soria y Valladolid, respectivamente) ${ }^{95}$.

Mención aparte merece el EA de Aragón, que admite una distorsión máxima de 1 a 2,75, como límite a la ley electoral, y de nuevo el EA de Valencia, que señala que la desproporción ha de ser inferior a la relación a 1 a 3. Y es obligada también una mención especial a las regulaciones provisionales de los EEAA de Cataluña y Canarias (reparto directo de escaños en las disposiciones transitorias respectivas), con unos índices de desviación de 1 a 2,2 en Cataluña (entre Lérida y Barcelona) y de 1 a 13,5 (i!) en Canarias (entre El Hierro y Tenerife) ${ }^{96}$.

e) La elevada barrera electoral, de carácter alternativo, que estableció la reforma del EA de Canarias de 1996, mediante la modificación de su disposición transitoria primera, y que estuvo vigente hasta la aprobación del EA de 2018: bien mayoría de votos o $30 \%$ en la circunscripción, bien $6 \%$ de votos en toda la comunidad. Son cifras descomunales, sin parangón en nuestro Derecho y en prácticamente todo el panorama comparado, que parecen de muy difícil justificación ${ }^{97}$.

Efectivamente, no parece que los argumentos de prudencia política, no constitucionalizados o estatutarizados expresamente, como la funcionalidad del Parlamento, la estabilidad del Gobierno o un sistema de partidos con fuerte implantación, constituyan suficiente fundamento para el menoscabo que, en su conjunto, se produce de derechos y principios constitucionales tan relevantes, como el pluralismo político, la igualdad del sufragio, la proporcionalidad de la representación, el acceso en condiciones de igualdad a los cargos públicos o, incluso, la adecuada representación de la población de los territorios ${ }^{98}$. No hay,

electoral, que refleja la divergencia total entre la distribución de escaños que resulta de la ley (la real) y la distribución que correspondería en función de la población (la teórica).

93 STC 225/1998, de 25 de noviembre, Fj 4, y ATC 240/2008, de 22 de julio, Fj 2.

94 En Andalucía el índice de distorsión del voto sube todavía más con la aplicación de la ley electoral que establece un mínimo provincial elevado de ocho escaños (artículo 17.2).

95 Todos los datos mencionados en este apartado son de elaboración propia, a partir de las cifras actualizadas de población.

96 Con la regulación vigente hasta 2018, la desproporción superaba la relación 1 a 17.

97 La doctrina se ha mostrado, por lo general, muy crítica en este punto. Vid. por todos López AGUILAR, J. F., «Los problemas de...», op. cit., pp. 123 y ss.

98 Algunos autores advierten, por otra parte, de las motivaciones políticas de la barrera establecida. Vid., entre otros, Oliver Araujo, J., Las barreras electorales..., op. cit., pp. 134-138. 
pues, proporcionalidad, en el sentido jurídico general, de justo equilibrio de los intereses en juego.

f) Las reformas del sistema electoral del EA de Castilla-La Mancha de 2007 y 2014, hechas sin consenso, y, la segunda, además, en la cercanía de un proceso electoral, aunque lo único que hizo en este punto fue retornar al sistema vigente hasta $1997^{99}$, el original de 1982, que sí reunió en su momento el consenso de los partidos. La exposición de motivos de la reforma es muy expresiva: «la presente propuesta de reforma pretende devolver a Castilla-La Mancha a la regulación anterior que supone una mejor adaptación del marco de la elección de los diputados regionales, al permitir una adaptación de la ley electoral correspondiente sin estar constreñida al rígido mandato estatutario» ${ }^{100}$.

No es ésta, desde luego, la mejor forma de proceder en esta materia, por el carácter central que el sistema electoral ocupa en las reglas que encauzan la legítima lucha partidista ${ }^{101}$. Las consecuencias sobre la legitimidad del sistema político, la estabilidad normativa e incluso las relaciones ordinarias entre las fuerzas políticas son muy negativas.

g) Finalmente, el mantenimiento de las regulaciones provisionales de los EEAA de Cataluña y Canarias, tras tantos años de andadura autonómica, por razones puramente partidistas. No hay más justificación, ciertamente, que el interés propio de las fuerzas beneficiadas, por el sistema vigente, en términos de resultados electorales, en que nada cambie: las nacionalistas en el primer caso y las coaliciones localistas en el segundo. Téngase presente, para entender el bloqueo, que, en ambas comunidades, la aprobación de la ley electoral que ha de sustituir dicha regulación provisional requiere una elevada mayoría: $2 / 3$ en Cataluña y $3 / 5$ en Canarias ${ }^{102}$.

\section{BREVE REFERENCIA A LA JURISPRUDENCIA CONSTITUCIONAL}

La segunda consideración adicional que anunciamos realizar, y con ella cerramos el estudio del tema, hace referencia a la jurisprudencia constitucional recaída sobre las disposiciones estatutarias relativas al sistema electoral autonómico. Se

99 Cuestión distinta es la reducción que también abordó, del número total de diputados, que es nueva.

100 Son las reformas del artículo 10.2 del EA de Castilla-La Mancha, producidas por la Ley Orgánica 3/1997, de 3 de julio, y la Ley Orgánica 2/2014, de 21 de mayo. Sobre estas reformas y las producidas en la Ley electoral vid. FERNÁNDEZ EsQuer, C., «Las sucesivas reformas del sistema electoral castellano-manchego: argumentos esgrimidos, motivaciones silenciadas», Revista Jurídica de la Universidad Autónoma de Madrid, nº 34, 2016, pp. 69-94; y Reviriego Picón, F., «Las reformas electorales y la magnitud de las circunscripciones», Estudios de Deusto, vol. 63/2, 2015, pp. 202 y ss.

101 Sobre la importancia del consenso en esta materia vid. Arnaldo AlCubilla, E., El carácter dinámico del régimen electoral, Madrid: CEPC, 2002, p. 18.

102 Vid., en la actualidad, los artículos 56.2 del EA de Cataluña y 39.2 del EA de Canarias. En esta última comunidad la reforma estatutaria de 2018 bajó la mayoría requerida desde 2/3 a 3/5. 
trata, más precisamente, de dar cuenta de la respuesta que el TC ha dado a los conflictos que le han llegado en este terreno, tras ponerse en cuestión formalmente en el plano jurídico la legitimidad constitucional de algunas de ellas.

Este examen ofrece una conclusión clara: el TC ha respaldado en todos los casos que se le han planteado las normas cuestionadas, siguiendo el criterio habitual de dejar un amplio campo de acción al poder legislativo en el ámbito del sistema electoral (general o autonómico), sentado en la primera sentencia sobre el tema, la 40/1981, de 18 de diciembre ${ }^{103}$. En este sentido el TC ha llegado a afirmar que «el pluralismo político y la libertad de configuración del autor de la ley son bienes constitucionales que debemos proteger» ${ }^{104}$. Este ejercicio de autocontención lo considera, además, especialmente pertinente en el caso de los EA, como integrantes del bloque de la constitucionalidad, «como un espacio abierto a distintas posibilidades legislativas» ${ }^{105}$.

No obstante, hay que precisar - lo que nos llevaría a una segunda conclusión- que no son muchos los casos suscitados ante la jurisprudencia constitucional y que los mismos están muy concentrados, en unos pocos temas y EEAA. Por tanto, no todo lo cuestionable o discutible de la regulación estatutaria del sistema electoral ha llegado al TC.

Conviene, por ello, dar cuenta de los elementos conflictivos sobre los que se ha pronunciado el TC para hacernos una idea más completa de la situación. Vamos a ser muy concisos en este punto, pues, en sí mismo, supone un campo de estudio propio, como prueba la realización de varios trabajos sobre resoluciones concretas $^{106}$. Vayamos por partes.

a) En buena parte de los recursos lo que se ha puesto en cuestión ha sido la legitimidad de la barrera electoral establecida, pero, como hemos anunciado, siempre sin éxito:

- La barrera electoral del 3\% de los votos emitidos, que establece de forma indirecta, por vía de remisión a la normativa estatal, la disposición transitoria primera del EA de Cataluña de 1989, aún aplicable (STC 75/1985, de 21 de junio).

103 Esta posición del TC ha recibido numerosas críticas en la doctrina. Vid., por ejemplo, ToRRES DEL Moral, A. y López Mira, A. J., «La jurisprudencia del Tribunal Constitucional en materia electoral. Acotaciones críticas», $R D P, \mathrm{n}^{\circ} 41,1996, \mathrm{pp} .18$ y 21.

104 STC 197/2014, de 4 de diciembre, Fj 5.

105 Vid. SSTC 75/1985, de 21 de junio, Fj 5; y 197/2014, de 4 de diciembre, Fj 7.

106 Así, por ejemplo, Garrote De Marco, M., «El control de constitucionalidad del sistema electoral de Castilla-La Mancha: a propósito de las SSTC 197/2014 y 15/2015», Revista de la Asamblea de Madrid, no 32, 2015, pp. 279-300; PÉrez Alberdi, M. R.: «Efectos de las barreras electorales: estudio del sistema electoral canario a raíz de la STC 225/1998», REP, n 52, 2001, págs. 357 y ss.; y López AguiLAR, J. F., «¿Control de constitucionalidad del Derecho Electoral Autonómico? Acerca dela STC 225/1998, sobre el sistema electoral canario (de nuevo sobre la «insoportable permanencia» de una disposición transitoria)», Repertorio Aranzadi del Tribunal Constitucional, 1, 1999, 1791-1804. 
- La barrera del 20\% de los votos emitidos en la circunscripción electoral, la isla, establecida en el art. 8.2 del EA de Canarias de 1982, en su versión inicial, como alternativa a la exigencia del $3 \%$ a nivel regional (STC 72/1989, de 20 de abril).

- En el mismo EA, pero ahora en la disposición transitoria primera, 2, en la redacción vigente entre 1996 y $2018^{107}$, la barrera electoral referida a las «listas de partido o coalición que hubieran obtenido el mayor número de votos válidos de su respectiva circunscripción electoral y las siguientes que hubieran obtenido, al menos, el 30 por 100 de los votos válidos emitidos en la circunscripción insular o, sumando los de todas las circunscripciones en donde hubiera presentado candidatura, al menos, el 6 por 100 de los votos válidos emitidos en la totalidad de la Comunidad Autónoma» (STC 225/1998, de 25 de noviembre).

En todos los casos la argumentación del TC es, más o menos, la misma. En primer lugar, rechaza que con las barreras se infrinja el principio de igualdad desde la perspectiva del artículo 23.2 de la CE en relación con el artículo 14, pues la regla legal se aplica a todas las candidaturas por igual: «Debe afirmarse, por el contrario, que el principio democrático de la igualdad se encuentra abierto a las fórmulas electorales más diversas, y ello porque se trata de una igualdad en la Ley, o, como el mismo art. 23.2 de la Constitución establece, de una igualdad referida a las «condiciones» legales en que el conjunto de un proceso electoral se desarrolla, por lo que la igualdad, por tanto, no prefigura un sistema electoral y excluye otros, sino que ha de verificarse dentro del sistema electoral que sea libremente determinado por el legislador, impidiendo las diferencias discriminatorias, pero a partir de las reglas de tal sistema, y no por referencia a cualquier otro» ${ }^{108}$.

$\mathrm{Y}$, en segund término, tampoco se infringe para el TC con dichas barreras el artículo 23.2 de la CE en conexión con el principio de proporcionalidad exigido por la CE y los EA para las elecciones autonómicas, por varias razones:

- Primero, la proporcionalidad no es «algo perfectamente delimitable, de manera unívoca, en todos sus contornos», sino «una orientación o criterio tendencial, porque siempre, mediante su puesta en práctica, quedará modulada o corregida por múltiples factores del sistema electoral» ${ }^{109}$.

- Segundo, la finalidad de esas barreras electorales es la de procurar que «la representación de los electores en tales Cámaras no sea en exceso fragmentaria, quedando encomendada a formaciones políticas de cierta relevancia», finalidad que es «de validez constitucional» «si tenemos en cuenta que el proceso electoral en su conjunto, no es sólo un canal para ejercer

107 Establecida por la Ley Orgánica 4/1996, de 30 de diciembre.

108 STC 75/1985, de 21 de junio, $\mathrm{Fj} 4$

109 STC 75/1985, de 21 de junio, Fj 5. 
derechos individuales (personales o de grupo) reconocidos por el art. 23 C.E., sino que es también, a través de esta manifestación de derechos subjetivos, un medio para dotar de capacidad de expresión a las instituciones del Estado democrático y proporcionar centros de decisión política eficaces y aptos para imprimir una orientación general de la acción de aquél» ${ }^{110}$.

- Tercero, »no es, por lo tanto, ilegítimo que el ordenamiento electoral intente conjugar el valor supremo que, según el art. 1.1 de la C.E., representa el pluralismo - y su expresión, en este caso, en el criterio de la proporcionalidad - con la pretensión de efectividad en la organización y actuación de los poderes públicos» ${ }^{111}$.

- Y cuarto, los límites establecidos no son exorbitantes ni contrarios al sistema de representación proporcional, pues se proyectan «sobre un sector relativamente reducido de los ciudadanos» ${ }^{112} \mathrm{y}$, además, en el caso canario, resultan adecuados «a las peculiaridades geográficas y poblacionales del archipiélago» ${ }^{113}$ y no hacen perder por sí solos al sistema canario su carácter proporcional» ${ }^{114}$.

Este último punto de su argumentación del TC es, sin duda, el más débil en el caso canario. Las barreras establecidas en el pasado en el EA, especialmente las de 1996, nos parecen desmedidas y poco proporcionales, como ya dejamos apuntado en el apartado anterior, opinión, por otra parte, bastante extendida en la doctrina ${ }^{115}$.

b) Otro tema conflictivo se ubica en el terreno del sistema de fuentes, pues se refiere a la propia forma de abordar la regulación del sistema electoral en el EA. En este punto son dos los elementos cuestionados, y que también han sido respaldados por el TC (STC 225/1998, de 25 de noviembre). Los dos estaban en el EA de Canarias y fueron introducidos por la reforma de $1996^{116}$ : el uso de una disposición transitoria del EA para regular diversos elementos del sistema electoral con una vigencia temporalmente incondicionada, hasta que sean regulados por una ley del Parlamento; y en estrecha conexión con ello, la exigencia de una mayoría cualificada (de 2/3) para aprobar la ley que establezca dichas reglas.

Sobre el primer punto el TC considera que «el grado de acierto del legislador acerca de la naturaleza temporal o definitiva de la disposición legal impugnada y su calificación como norma de derecho transitorio no es per se elemento

110 Ibidem.

111 Ibidem.

112 STC 225/1998, de 23 de noviembre, fj 5.

113 STC 72/1989, de 21 de junio, Fj 3.

114 STC 225/1998, de 23 de noviembre, fj 7.

115 Vid., por ejemplo, Torres del Moral, A., «Sistemas electorales y...», op. cit., pp. 232-234; y López Aguilar, J. F., «¿Control de constitucionalidad...», op. cit., pp. 1791 y ss.

116 Se establecía en su DT $1^{\text {a }} 1$, que decía así en su comienzo: «De acuerdo con lo establecido en el artículo 9 del presente Estatuto, y en tanto no se disponga otra cosa por una Ley del Parlamento Canario aprobada por mayoría de dos terceras partes de sus miembros...» 
condicionante de su constitucionalidad, ni supone en su sola consideración individual, una quiebra del principio de seguridad jurídica reconocido en el art. 9.3 $\mathrm{CE}$ », que en todo caso el precepto estatutario «es perfectamente claro y no ofrece especiales dificultades de comprensión y entendimiento que puedan inducir a error o confusión» y que deferir al legislador autonómico el contenido definitivo de la regulación «no comporta abrir el Estatuto a un inconstitucional proceso de reforma, sino efectuar una atribución constitucionalmente posible». Y en cuanto al segundo, tras apuntar que estamos ante una materia en la que "por su propia naturaleza», cabe «justificar la sustracción a la regulación por mayoría simple», afirma que «ningún reparo puede existir para que el Estatuto de Autonomía de esa Comunidad Autónoma pueda, en tanto que «norma institucional básica» (art. 147.1 CE) y, por tanto, norma sobre la producción del derecho propio de la Comunidad Autónoma, imponer una mayoría en orden al ejercicio de la competencia legislativa autonómica sobre la materia» ${ }^{117}$.

c) Fuera del ámbito de la barrera electoral y de la configuración de la regulación del sistema electoral por el propio EA solo hay una cuestión que haya sido objeto de recurso ante el TC: la reducción del tamaño del Parlamento por medio de la reforma del EA de Castilla-La Mancha de 2014, pasando de una horquilla de 47 a 59 diputados a otra de 25 a 35, sin que tampoco en este caso el máximo intérprete de nuestra norma suprema haya encontrado tacha alguna de inconstitucionalidad (STC 197/2014, de 4 de diciembre) ${ }^{118}$, decisión al menos cuestionable por las razones apuntadas anteriormente ${ }^{119}$.

El TC considera evidente, por un lado, que «la autonomía política de la Comunidad Autónoma [arts. 2 y $137 \mathrm{CE}$ ] y su consiguiente capacidad de legislar en los ámbitos de su competencia [art. 152.1 CE y art. 9.2.a) EACM] no queda empañada, ni afectada siquiera, por la reducción del número de miembros de la Cámara autonómica que ha llevado a cabo la Ley» ${ }^{120}$, y por otro lado, que aunque esta reducción «no favorece, más bien al contrario, la mayor proporcionalidad del sistema electoral», no se quiebra la «esencia de la proporcionalidad» ${ }^{121}$. En relación con este punto, y sobre el concreto reproche de la mayor dificultad para conseguir escaño en las distintas circunscripciones, afirma: «Se trata de unos hipotéticos umbrales porcentuales para la obtención, según los varios «escenarios» que anticipan, del último escaño en liza conforme a la regla d'Hondt y ello sobre la base de estimaciones conjeturales, ajenas a cualquier determinación

117 STC 225/1998, de 25 de noviembre, $\mathrm{Fj} 2$.

118 El TC también rechazó el recurso contra la posterior reforma de la Ley electoral de Castilla-La Mancha (Ley 142014, de 21 de julio) que concretó el número de diputados en 33 (STC 15/2015, de 5 de febrero).

119 Vid. también Fernández Esquer, C., «La reforma del sistema electoral de Castilla-La Mancha de 2014», Cuadernos Manuel Giménez Abad, no 11, pp. 78 y ss.

$120 \mathrm{Fj} 3$.

$121 \mathrm{Fj} 7$ 
excluyente del legislador y dependientes más bien, como prospecciones o simulaciones que son, del número de candidaturas que lleguen a concurrir y de la mayor o menor concentración o dispersión sobre ellas del futuro sufragio de los electores» ${ }^{122}$.

Finalmente, antes de terminar, es conveniente aludir a varias resoluciones sobre recursos constitucionales en los que lo que se cuestiona no es una disposición estatutaria, sino de una ley electoral autonómica, y ello en la medida en que existen o han existido disposiciones parecidas en los EEAA, a las que, por tanto, cabría extender las decisiones adoptadas. También aquí el aval del TC ha sido la tónica:

- La STC 193/1989, de 16 de noviembre, que admite la legitimidad constitucional de una barrera electoral del $5 \%$ de los votos válidos emitidos a nivel de toda la CA y no de la circunscripción ${ }^{123}$. Tiene interés para nosotros, porque, hasta 2006, existía, como sabemos, una disposición parecida en el EA de Valencia.

- El ATC 240/2008, de 22 de julio, que avala la falta de corrección por el legislador de las distorsiones en el reparto de escaños entre las circunscripciones producidas por la distinta evolución de los flujos de población, con la apostilla de que «una prolongada inacción del legislador, consintiendo durante períodos excesivos alteraciones significativas que desvirtúen la proporcionalidad de la atribución de escaños puede llegar a provocar la inconstitucionalidad sobrevenida de la norma que establezca la distribución provincial» ${ }^{124}$. Es una resolución cuya doctrina es trasladable a los EEAA que realizan un reparto fijo y directo de escaños, como sucede con las disposiciones transitorias de los EEAA de Cataluña y Canarias. Incluso cabría pensar en su aplicación al reparto igualitario de escaños del País Vasco.

- La STC 45/1992, de 2 de abril, que admite la compatibilidad con el principio de proporcionalidad electoral de un reparto de escaños entre las circunscripciones no ajustado al volumen de población de cada una de ellas, siempre que no exista — matiza el TC — una situación de «manifiesta y arbitraria desproporción» ${ }^{125}$. La doctrina que encierra esta sentencia resulta de aplicación a las numerosas disposiciones estatutarias que, de

122 Ibidem.

123 Artículo 15.a) de la Ley 2/1987, de 24 de febrero, Electoral de la Región de Murcia, según el texto anterior a la importante reforma de 2015 (Ley 14/2015, 28 julio).

124 El Auto se refiere al artículo 16.2 de la Ley 5/1986, de 23 de diciembre, electoral de Castilla-La Mancha, en la redacción dada por la Ley 8/1998.

125 Esta Sentencia respalda la disposición de la Ley electoral balear que contenía una clara distorsión en el prorrateo (Artículo 12.2 de la Ley 8/1986, de 26 de noviembre), pues a una circunscripción con menos población que otra se le otorgaba un escaño más (Menorca frente a Ibiza). Vid. Fernández Esquer, C., «Algunos problemas en...», op. cit., pp. 337-340; y Oliver Araujo, J., Los sistemas electorales autonómicos..., op. cit., pp. 261-264. 
alguna forma, establecen o permiten un prorrateo desviado de escaños, es decir, una distribución de escaños con una distorsión significativa sobre el valor población.

$$
* * *
$$

TITLE: Statutes of autonomy and autonomic electoral systems.

ABSTRACT: This paper deals with examining the confluence between the statute of autonomy and the electoral system, as maximum exponents of two fundamental sectors of Spanish constitutional law: the autonomous regime and the electoral regime. For this purpose, the content of the statutes on the autonomous electoral system is realized, its importance is calibrated, the contributions made to the electoral law as a whole, especially Spanish, are individualized, and it is concluded, finally, with an assessment of the regulation and a brief reference to the position of the Constitutional Court on the few conflicts raised.

RESUMEN: Este trabajo se ocupa de examinar la confluencia entre el estatuto de autonomía y el sistema electoral, como exponentes máximos de dos sectores fundamentales del Derecho constitucional español: el régimen autonómico y el régimen electoral. A este propósito, se da cuenta del contenido de los estatutos sobre el sistema electoral autonómico, se calibra su importancia, se individualizan las aportaciones realizadas al conjunto del Derecho electoral, en especial el español, y se concluye, finalmente, con una valoración de la regulación y una breve referencia a la posición del Tribunal Constitucional sobre los escasos conflictos planteados.

KEY WORDS: Electoral law, autonomous law, autonomy statute, electoral system, electoral reform, autonomous parliaments, elections, autonomous communities, electoral constituency, electoral barrier, proportionality.

Palabras Clave: Derecho electoral, Derecho autonómico, estatuto de autonomía, sistema electoral, reforma electoral, parlamentos autonómicos, elecciones, comunidades autónomas, circunscripción electoral, barrera electoral, proporcionalidad.

FECHA DE RECEPCIÓN: 28.01.2020

FeCHa DE ACEPTACIÓN: 19.02.2020 
